LUBLIN STUDIES IN MODERN LANGUAGES AND

LITERATURE 40(1), 2016, HTTP://WWW.LSMLL.UMCS.LUBLIN.PL, HTTP://LSMLL.JOURNALS.UMCS.PL

\author{
Haniyeh Barahouie \\ Université de Virginie \\ Cabell Hall, $\mathrm{N}^{\circ} 321$ \\ 22904, VA, États-Unis
}

\title{
Le dédoublement réaliste-minimaliste des récits contemporains français : Le cas de Je m'en vais de Jean Echenoz et de Fuir de Jean-Philippe Toussaint
}

\begin{abstract}
In the mid-1980s, a number of contemporary French writers attempted to renew the genre of the French novel through a multilayered stylistic revival. The critics had condemned the novel to death as it was besieged with theories and did not offer anything new. The new generation emphasized on writing and ignored all other theory-based approaches. The critics began identifying the new phenomena and ultimately came up with the "minimalist" label for writers of this clan. The novels of Jean Echenoz and Jean-Philippe Toussaint are remarkable examples of this literary movement as they illustrate all aspects of minimalist art in I'm Off (Je m'en vais) and Running away (Fuir). This paper deals with the resemblances and the differences between minimalism and realism. It will also highlight the traces of realism in contemporary writings in order to show how well its components are maintained or rejected in the new literary texts.

Keywords: Jean Echenoz; Jean-Philippe Toussaint; contemporary novel; minimalism; realism.
\end{abstract}


La première occurrence de la notion de minimalisme fut aux ÉtatsUnis dans les années soixante, à travers les appellations de «minimal music » et de «minimal art». Par la suite, elle a été insérée dans les années quatre-vingt, dans la littérature américaine. L'idée de minimalisme est venue en France au début de cette même décennie. La notion de roman «minimaliste » est étiquetée par la critique à une collection d'auteurs affiliés aux Éditions de Minuit.

Notre choix inclut les ouvrages clés de Jean Echenoz et JeanPhilippe Toussaint étant, selon le mot de Fieke Schoots, « le plus souvent tenus responsables du renouveau romanesque [des années 1980]» (1997 : 24). Ce compte rendu s'explique au gré des qualités minimalistes intrinsèques qui existent entre Je m'en vais et Fuir.

Fiecke Schoots dans son Passer en douce à la douane traduit l'ensemble des traits rapportés en termes de procédés narratifs étroitement associés au contenu narratif, à la forme et au style des œuvres. Étant donné qu'il n'a y a pas assez d'espace pour mentionner l'ensemble des traits minimalistes, on essayera d'y faire une allusion exhaustive.

Sur le plan du contenu, le minimalisme concerne l'intrigue, les personnages et les décors, ceux qui se dessinent dans l'univers romanesque des deux romans dressant en filigrane le portrait de la société contemporaine au gré d'un système des objets ainsi qu'une cartographie spatiale ${ }^{1}$. Dans Je m'en vais et Fuir, l'accent est mis plus sur le décor que sur l'action d'où une réduction de l'intrigue au minimum. Formellement, le minimalisme privilégie, de manière ascendante, la brièveté des mots, des phrases, des paragraphes et des récits. L'adaptation d'un style paratactique par ces deux auteurs démontre leur intérêt central porté à la figure brève de leur récit. Du point de vue stylistique, le récit minimaliste agit au niveau de la syntaxe, du vocabulaire et des figures utilisées. Malgré la banalité existant dans l'intrigue, Toussaint et Echenoz savent très bien manipuler le lecteur en vue d'éviter l'ennui. En tenant compte des

$1 \quad C f$., Christine, Jérusalem, «Géographies de Jean Echenoz », http://remue.net/spip.php?article3126, consulté le 27 novembre 2014. 
traits minimalistes de ces deux romans, où se place le réalisme ? La notion de réalisme peut-elle prendre un contrepied dans le récit minimaliste?

1. Le réalisme ou le minimalisme

La question du rapport des auteurs de Minuit au «réel » n'a pas cessé d'occuper une partie des débats critiques. En ce sens, il est essentiel de revenir rapidement sur l'esthétique réaliste en vue d'examiner ses filatures dans les récits d'Echenoz et de Toussaint. En matière de réalisme, il existe une attitude de l'artiste face au réel, qui a pour ambition de représenter le plus fidèlement possible la réalité telle qu'elle est. Du réalisme, Echenoz et Toussaint empruntent le goût pour les objets, le décor et les descriptions pointilleuses. Nous prenons l'exemple du Flétan: le « jeune homme aux yeux fermés [qui] aurait même l'air un peu mort » (Echenoz 1999: 82) qui habite dans un « gourbi » comme en écho à quelque pension Vauquer qu'on n'en finirait plus de décrire ou encore la description du wagon-restaurant donnant des détails à profusion autant sur l'espace que sur les plats :

Nous avions pris place dans le wagon-restaurant et commandé quelques plats, des brochettes, du porc au gingembre, des nouilles sautées. La nappe était tachée de traces de thé brunâtres et de sauce d'un précédent repas, des cendres débordaient d'une soucoupe remplie de mégots. (Toussaint 2009 : 30-31).

Pour dessiner les itinéraires des personnages, ils s'équipent d'une certaine richesse documentaire. Avant de se mettre à écrire $\mathrm{Je}$ m'en vais, Jean Echenoz s'est préalablement documenté sur le pôle Nord : la Nechilik, son lieu d'échouage, les œuvres d'art inuit, Port Radium, etc. Quant à Fuir, son auteur a fréquenté les grandes villes de la Chine afin de fournir une peinture autant que possible réaliste de sa figure contemporaine. Ce compte rendu de la réalité chez eux, nous penche sur une réflexion de fond: dans quelle mesure ce réalisme s'écarte de celui exigé par Maupassant et se rapproche du minimalisme ? 
2. Un réalisme déconcerté

En ce qui concerne le réalisme, l'écriture echenozienne et toussaintienne se rapprochent de celle de Maupassant d'autant plus que dans la préface de «Pierre et Jean», ce dernier explique la virtuosité du romancier, laquelle réside « dans le groupement adroit de petits faits d'où se dégagera le sens définitif de l'œuvre» (1909: 12). Selon Maupassant, il s'agit de prendre un personnage « à une certaine période de [son] existence » $(1909: 12)$ et de le conduire "par des transitions naturelles jusqu'à la période suivante» (1909 : 12), en démontrant comment les esprits se modifient sous «l'influence des circonstances environnantes » (1909: 12). Chez l'un aussi bien que chez l'autre écrivain, de nombreux éléments thématiques et formels, viennent dérégler ce modèle. Sur le plan thématique, bien que la continuité soit respectée, les personnages de Je m'en vais et de Fuir ne sont pas nettement définis dans la société, parallèles au décor, ils se caractérisent au gré des objets. Le décrochage narratif, la chronologie non respectée surtout dans Je m'en vais et l'intrigue réduite au minimum sont autant d'enjeux pour déstabiliser le lecteur traditionnel et déroger au réalisme. Le narrateur est censé d'être autant limpide et discret que possible: «il devra donc composer son œuvre d'une manière si adroite, si dissimulée, et d'apparence si simple, qu'il soit impossible d'en apercevoir et d'en indiquer le plan, de découvrir ses intentions » selon les paroles de Maupassant (1909: 12).

Tel n'est pas le cas dans Je m'en vais et Fuir, là où le narrateur introduit son propre avis en ouvrant un paragraphe ou une parenthèse pour déjouer son rôle d'énonciateur aussi bien que brouiller la linéarité du récit. Le recours apparent à un flux narratif formé autour d'une intrigue quasi inhérente, aux personnages introduits dans le monde contemporain et à un narrateur/énonciateur, ne constitue qu'un « effet de réel » (Schoots 1997 : 162) selon Roland Barthes.

3. Le minimalisme des deux romans

«Les deux esthétiques - réalisme et minimalisme - sont naturellement en opposition forte: le réalisme est saturant, il prétend à l'exhaustivité, le minimalisme au contraire fait l'épreuve de la 
réduction la plus sévère » (Viart $2004: 20$ ). On verra qu'avec le choix de telles histoires, celles d'une fuite, les auteurs se donnent la possibilité d'appliquer ces deux esthétiques dans leurs récits. La gratuité de l'intrigue (l'intrigue policière parodiée par Echenoz ainsi que l'histoire amoureuse du narrateur de Fuir où il ne se passe pas grand-chose) et la rupture paratextuelle par des larges marges ont donné une dimension minimaliste aux récits. Il en va de même avec le déficit communicationnel (Jérusalem 2007 : 68-70) dans les deux romans :

Je crois que ça avance avec Nechilik, dit Delahaye. La quoi ? dit Ferrer. Le bateau, là, dit Delahaye, vous savez, le bateau des antiquités. Je crois que j'ai trouvé des informateurs. Ah oui, dit Ferrer évasivement, distrait par le grelot de la porte d'entrée. Attention, souffle-t-il, quel qu'un. Réparez (Echenoz 1999 : 4041).

Ce discours rapporté, évacué de ponctuation adéquate, est réduit au minimum. Tel est le cas du narrateur de Fuir dont les dialogues sont automatiquement diminués à cause de la perte du canal communicationnel lequel est la langue chinoise.

Pour mieux comprendre les dimensions minimalistes des deux récits, il faut les voir sous un autre angle : celui des scènes décisives. Or, pensons au dénouement policier de Je m'en vais, à la rencontre hasardeuse de Ferrer et Delahaye. Le narrateur se contente de quelques dialogues entre les deux personnages pour en fournir son aparté ludique tout au milieu de la querelle :

Cependant que l'autre, tête versée en arrière au-dessus du fleuve bouillonnant, après avoir tentée d'en mettre aussi mêlées de protestation, ne gargouillait plus que non, non, je vous en prie, non.

Nous n'avons pas pris le temps, depuis presque un an pourtant que nous le fréquentons, de décrire Ferrer physiquement. Comme cette scène un peu vive ne se prête pas à une longue digression, ne nous $\mathrm{y}$ éternisons pas (Echenoz 1999 : 234).

Cette tonalité volontairement impassible, cette superficialité descriptive et notamment ce commentaire dérisoire dévalorisent la scène de la dispute traditionnelle en lui donnant la figure d'un plateau banal. 
De la même façon, l'histoire de Fuir manque délibérément l'inhérence narrative dans les scènes clés par des lignes de fuites. Le passage du deuxième au troisième chapitre du livre, sans jamais donner une conclusion au chapitre chinois, en fournit l'exemple éloquent. Après avoir quitté les deux Chinois dans la rue au deuxième chapitre du roman, le narrateur ignore leurs destins en disant :

De retour à l'hôtel, à Pékin, [...], je n'avais entendu aucun bruit dans leur chambre, de sorte que je ne sais toujours pas s'ils sont jamais rentrés (Toussaint 2009: 122).

$\mathrm{Au}$ cours de trois pages blanches, la scène décisive de la fuite des trois personnages détourne le lecteur habituel par sa figure à la fois brève et laconique associée au tournant de l'histoire.

Il faut tenir également compte du décor de l'art minimal dans $J e$ m'en vais dont les débats se sont placés dans les années quatre-vingtdix. S'installant dans le milieu des galeristes, les allusions aux problèmes financiers de la galerie de Ferrer, la recherche de l'art Inuit et principalement l'évocation des œuvres minimalistes (monochromes jaunes de Martinov, sommeils de Fracnatz, les installations de Guimard, etc.) font l'objet d'une représentation artistique minimaliste.

Pour en ainsi dire que «les récits "minimalistes" ne sont ni radicalement fragmentés, ni parfaitement continus. Ils sont fragmentaires malgré la narration d'une histoire continue ; ils sont cohérents malgré les blancs qui interrompent l'histoire» (Schoots 1997 : 139). Que retenir donc de ces rebondissements esthétiques ?

\section{Conclusion}

Ce que revendique le minimaliste c'est la production «sans travail de [la] forme ni de [la] matière » (Viart 2004: 25) de son œuvre. S'inscrivant dans la lignée de Duchamp, les minimalistes recourent au ready-made, aux matériaux industriels, auxquels ils ne font subir aucune modification. Cela dit, le rôle de l'artiste ainsi que sa simple décision de choisir et d'exposer ceci plutôt que cela sont effacés autant que possible. Il en va de même avec le réalisme qui "prétend se borner à une sélection de phénomènes, d'évènements ou d'objets, 
parmi ceux que la réalité lui offre à profusion » (Viart 2004 : 25). Par conséquent, la représentation de la réalité telle qu'elle est le point d'équivalence entre le réalisme et le minimalisme. Cependant, ce qui les différencie l'un de l'autre, s'enracine dans ce fait que « le réaliste a tendance à promouvoir la redondance, comme Philippe Hamon l'a montré dans «Un discours contraint », alors que le minimaliste au contraire essaie de réduire les éléments » (Viart $2004: 26$ ).

Echenoz et Toussaint jouent, sans doute, ces esthétiques à les déjouer. Quant au réalisme, ils désobéissent à une narrativité nette aussi bien qu'à l'exhaustivité et à la vraisemblance. Loin de copier une image fidèle de la réalité, ils essaient d'en créer et d'en produire. Leurs textes ne sont pas des transcriptions de la réalité, «mais des simulacres, c'est-à-dire des mondes tout à fait originaux créés à partir de quelques traits réalistes» (Schoots 1997: 178). Or, leur intervention dans la production artistique, leur goût de l'inventivité ainsi que leur souci d'une forme minutieusement travaillée les dissocient du minimalisme et installent leur texte dans un «espace indécidable» (Viart $2004: 24$ ).

\section{Bibliographie}

Blanckeman B. (2000) : Les récits indécidables. Jean Echenoz. Hervé Guibert. Pascal Quignard. Paris : Coll. «Perspectives », Presses Universitaires du Septentrion.

Blanckeman B. (2002) : Les fictions singulières. Paris : Prétexte Éditeur.

Blanckeman B. et Millois J-C. (2004): Le roman français aujourd'hui. Paris : Prétexte éditeur.

Bond D. (2000) : «Small Worlds: Minimalism in Contemporary French Literature by Warren Motte » French Forum, Vol. 25, N 2, University of Nebraska Press Stable, pp. 242-243, URL: http://www.jstor.org/stable/40552108 (consulté le 02 décembre 2014).

Echenoz J. (1999) : Je m'en vais. Paris : Éditions de Minuit.

Jérusalem Ch. (2007) : Je m'en vais de Jean Echenoz. Paris : Hatier.

Jérusalem Ch. (2007) : «Géographies de Jean Echenoz», http://remue.net/spip.php?article3126, (consulté le 27 novembre 2014).

Lebrun J.C et Prévost C. (1990): «Jean Echenoz, l'accélérateur de particules» Nouveaux territoires romanesques. Messidor Éditions Sociales, pp. 95-110.

Lebrun J.C et Prévost C. (1992) : Jean Echenoz. Paris : Édition du rocher. 
Maupassant G. (1909) : Pierre et Jean. Paris : Conard.

Mitterend H. (1994) : «L'Illusion réaliste de Balzac à Aragon », P.U.F, Coll. « Écriture», pp. 63-64.

Panaïté O. (2006) : «Les fins de l'écriture. Réflexion et pratique du style dans les œuvres de Jean Echenoz et Pierre Michon », French Forum, Vol. 31, N ${ }^{\circ} 2$, University of Nebraska Press Stable, pp. 95-110. URL: http://www.jstor.org/stable/40552433 (consulté le 07 décembre 2014).

Shoots F. (1997) : Passer en douce à la douane. L'Écriture minimaliste de Minuit: Deville, Echenoz, Redonnet, Toussaint. Amsterdam, Atlanta : Rodopi.

Toussaint J-P. (2009) : Fuir. Paris : Éditions de Minuit.

Viart D. (1993) : «Le récit 'postmoderne' », La littérature française contemporaine, questions et perspectives, Presses Universitaires de Louvain, pp.153-165.

Viart D. (2004) : «les esthétiques démenties », Revue du roman du XXe siècle, N॰38, Lille, Presse de l'université de Charles-de-Gaulle, pp. 14-26. 